\title{
Corela
}

Cognition, représentation, langage

HS-31 | 2020

Métalinguistiques.

\section{Analyse des comportements épilinguistiques et des activités métalinguistiques des élèves dans les disciplines non-linguistiques des écoles bilingues songhay-français}

\section{Zakaria Nounta}

\section{(2) OpenEdition}

\section{Journals}

Édition électronique

URL : http://journals.openedition.org/corela/11687

DOI : 10.4000/corela. 11687

ISSN : $1638-573 \mathrm{X}$

Éditeur

Cercle linguistique du Centre et de l'Ouest - CerLICO

Référence électronique

Zakaria Nounta, «Analyse des comportements épilinguistiques et des activités métalinguistiques des élèves dans les disciplines non-linguistiques des écoles bilingues songhay-français », Corela [En ligne], HS-31 | 2020, mis en ligne le 02 juillet 2020, consulté le 03 juillet 2020. URL : http:// journals.openedition.org/corela/11687 ; DOI : https://doi.org/10.4000/corela.11687

Ce document a été généré automatiquement le 3 juillet 2020

\section{(c) (i) (3)(2)}

Corela - cognition, représentation, langage est mis à disposition selon les termes de la licence Creative Commons Attribution - Pas d'Utilisation Commerciale - Partage dans les Mêmes Conditions 4.0 International. 


\title{
Analyse des comportements épilinguistiques et des activités métalinguistiques des élèves dans les disciplines non-linguistiques des écoles bilingues songhay-français
}

\author{
Zakaria Nounta
}

\section{Introduction}

1 Le métalinguistique relève de la capacité que le langage, à travers l'ensemble de ses activités, a de renvoyer à lui-même. Il se réfère à la réflexion et à la manipulation des caractéristiques structurales du langage dont le caractère conscient et délibéré a pu être établi. (Demont, Gaux \& Gombert 2006).

Dans le contexte de l'enseignement-apprentissage bilingue où les élèves sont sensibilisés aux spécificités de l'univers du langage à partir d'un certain nombre d'activités d'observation et de réflexion portant sur des faits de langue, la question qui se pose est de savoir si l'élève réfléchit consciemment sur le langage.

Pour expliquer les comportements cognitifs vis-à-vis du langage, Gombert (1990) propose de classer les activités de type métalinguistique en deux catégories : les activités épilinguistiques et les activités métalinguistiques. Les premières désignent les comportements métalinguistiques qui montrent la " maîtrise fonctionnelle des règles d'organisation ou d'usage de la langue » (Gombert 1990 : 27) de l'individu, mais qui ne sont pas contrôlés consciemment par celui-ci. Les secondes se réfèrent aux activités réfléchies et délibérées du sujet qui portent sur le langage et son utilisation.

De nombreux auteurs (notamment Candelier 2003 ; Werner 1949 ; Kanta et al. 2006) ont mis en évidence les effets positifs du bilinguisme sur le développement de la conscience métalinguistique. Ils affirment que l'acquisition d'un lexique en L1 et en L2 développe 
les capacités méta-discursives sur les mots. En outre, le bilinguisme précoce favorise le développement cognitif à plusieurs niveaux, bien au-delà du langage (Bialystok et al. 2012), ce qui se reflète surtout dans les disciplines comme le calcul ou les sciences expérimentales. Enfin, il convient d'observer les comportements métalinguistiques dans les classes bilingues pour des domaines d'enseignement autres que les langues, moins contraints par les programmes et les modes d'entraînement portant sur le langage. De ce fait, les situations d'enseignement dans les disciplines non-linguistiques sont les mieux indiquées pour étudier chez les élèves bilingues le développement du langage en deux langues (Noyau 2014).

5 Parallèlement, il a été établi par d'autres travaux (Belloncle 1984 ; Doumbia 2000) que la difficulté majeure liée aux systèmes éducatifs dans la plupart des pays d'Afrique francophone demeure le monolinguisme scolaire. Ces auteurs postulent que l'utilisation exclusive du français, langue totalement inconnue des enfants entrant à l'école, peut avoir des conséquences négatives. Ces études ont influencé plusieurs états francophones d'Afrique dont le Mali, et les ont amenés à l'enseignement bilingue. Mais, dans ces pays, et particulièrement au Mali, les insuffisances dans la formation des maîtres amenuisent leurs capacités à mener une réflexion métalinguistique sur les langues. N'étant pas formés à travailler le niveau métalinguistique, les enseignants se contentent uniquement de ce qui est inscrit dans les manuels, concernant le domaine d'enseignement Langage.

6 Dans ce contexte, comment se manifestent les comportements épilinguistiques et les activités métalinguistiques des élèves dans les disciplines non-linguistiques?

7 À partir des résultats issus du codage de six enregistrements vidéo, en mathématiques et en sciences d'observation, effectués dans les classes de 2ème, 4ème et 5ème années fondamentales d'une école bilingue songhay-français, nous avons étudié les différentes manifestations des comportements épilinguistiques et des activités métalinguistiques des élèves.

\section{Le contexte du système éducatif du Mali : la scolarisation bilingue à travers l'approche curriculaire bilingue par compétences}

8 À l'instar de plusieurs pays africains, le Mali s'est ouvert à une conception de l'école faisant place à l'utilisation des langues nationales dans l'enseignement, en prescrivant un enseignement bilingue basé sur l'approche curriculaire par compétences. Celle-ci favorise une nouvelle vision de l'apprentissage où l'apprenant est artisan de sa propre formation et travaille régulièrement à résoudre des problèmes et tâches intégratives éliminant ainsi les barrières entre les disciplines.

9 Mais le choix de cette approche requiert la mise en place d'une pédagogie qui puisse organiser les transferts de connaissance et la mise en relation entre L1 et L2, ce qui nécessite une bonne formation des maitres.

10 Or, au Mali, la qualification du personnel enseignant est un sujet délicat. En effet, la mise en œuvre de la politique de décentralisation a introduit de nouvelles pratiques dans la gestion de l'école et particulièrement dans le recrutement, la formation et la gestion du personnel enseignant. 
11 Conjointement à cette situation, il y a eu un accroissement rapide de la population scolaire qui a entraîné un développement des initiatives privées et communautaires dans les créations d'écoles.

12 C'est dans ce contexte que plusieurs innovations pédagogiques ont été initiées en vue d'améliorer les pratiques d'enseignement. Parmi ces innovations, l'introduction de onze langues nationales sur les treize reconnues par la loi dans l'enseignement formel à travers d'abord la pédagogie convergente, puis l'approche curriculaire par compétences.

13 Dans les classes de $1^{\text {ère }}$ année du primaire, l'enseignement s'effectue exclusivement en langue première de l'enfant. Le temps d'enseignement est subdivisé en deuxième année : $75 \%$ du temps est consacré à l'enseignement en L1 et $25 \%$ en L2. En troisième et quatrième années, le rapport s'inverse et $75 \%$ de l'horaire sont réservés à l'apprentissage du français (oral et écrit) et $25 \%$ aux apprentissages en L1. En cinquième et sixième années, la répartition du volume horaire est équitable : $50 \%$ pour la L1 et $50 \%$ pour la L2.

14 Même s'il existe à ce jour partout dans le pays une majorité d'écoles dites " classiques " dans lesquelles seul le français demeure la langue d'enseignement, l'éducation bilingue concerne tout de même un nombre significatif d'établissements primaires.

\section{Définition des concepts}

\subsection{Concept d'épilinguistique}

15 La notion d' «épilinguistique » a été créée par le linguiste Culioli (1968). Elle a été utilisée pour désigner des activités proches du comportement métalinguistique mais qui sont réalisées sans contrôle conscient. Les activités épilinguistiques sont donc définies comme étant celles qui échappent au contrôle de la conscience: "comportements précocement avérés qui s'apparentent aux comportements métalinguistiques mais qui ne sont pas contrôlés consciemment par le sujet. Il s'agit en fait de manifestations explicites, dans les comportements des sujets, d'une maîtrise fonctionnelle de règles d'organisation ou d'usage de la langue.» (Gombert 1990: 27)

16 Les activités épilinguistiques concernent les comportements portant sur le langage, non délibérés, manifestés par hésitations, répétitions, reprises et reformulations. Elles consistent en une "connaissance intuitive et un contrôle fonctionnel des traitements linguistiques» (Gombert 1990:233). Dans ce cas, le sujet déploie fréquemment une activité de contrôle sur sa production qui s'exerce par exemple sur les règles grammaticales implicitement utilisées. Les comportements épilinguistiques peuvent être observés notamment à l'occasion des phénomènes d'autocorrection.

17 L'enfant applique inconsciemment des règles dont il ignore le fonctionnement.

18 Dans notre travail de recherche, les comportements épilinguistiques correspondent aux hésitations, aux répétitions et aux reprises avec reformulation de l'élève.

\subsection{Concept de métalinguistique}

Le concept de métalinguistique se définit comme l'adoption d' " une attitude réflexive sur les objets langagiers et leur manipulation» (Gombert 1990: 11). Il est lié à la métalangue, 
terme qui désigne la langue ayant pour lexique l'ensemble des mots de la terminologie linguistique, et dont l'unique but est de parler d'une langue. Le métalangage a pour principale fonction la description d'une langue.

Selon Gombert (1990), l'acception du terme «métalinguistique » varie selon qu'on est psycholinguiste ou linguiste. La définition psycholinguistique du terme «métalinguistique » est plus large que celle attribuée par la linguistique : «le linguiste dépistera "le métalinguistique» en identifiant dans des productions verbales des marques linguistiques traduisant des processus d'autoréférenciation (utilisation du langage pour référer à lui-même). Le psychologue, quant à lui, cherchera dans le comportement (verbal ou non) du sujet des éléments qui lui permettront d'interférer des processus cognitifs de gestion consciente (de réflexion sur, ou de contrôle délibéré), soit des objets langagiers en tant que tels, soit de leur utilisation.» (Gombert 1990: 15).

Dans notre étude, le métalinguistique désigne la capacité que le langage a de renvoyer à lui-même à travers l'ensemble de ses activités. Les activités métalinguistiques concernent toutes les manipulations et réflexions conscientes portant sur le langage, permettant de s'en construire une représentation explicite. Ces manipulations et réflexions permettent de décrire le langage même ordinaire. Ainsi, nous considérons les reformulations et les définitions, même menées en langage ordinaire, comme des activités métalinguistiques. Le métalinguistique est un niveau d'activité qui ne relève pas seulement ou uniquement du métalangage ou de la métalangue, que nous considérons comme étant les utilisations autoréflexives du langage, qui dans ce cas, devient l'objet d'étude. Le métalangage et la métalangue sont donc une partie du métalinguistique. Ils relèvent de la verbalisation du métalinguistique.

\section{Fondements théoriques}

Nous nous sommes appuyé sur les théories de l'acquisition du bilinguisme et les fonctions des interactions pédagogiques.

Les théories relatives à l'acquisition du bilinguisme établissent que les langues utilisées par un bilingue font appel en apparence à des mécanismes séparés, mais en réalité, elles fonctionnent grâce au même système cognitif central : «lorsqu'une personne possède deux langues ou plus, il y a une unique source de pensée intégrée. » (Baker 1996: 147).

L'utilisation de certaines fonctions langagières autrement dit le développement lexical et conceptuel en L1 dans l'environnement de l'enfant, conduit nécessairement à un haut degré de compétence en L2, sans effets négatifs sur L1.

"Le haut niveau de compétence langagière atteint en L1 permet qu'un niveau équivalent soit atteint en L2. 》 (Cummins 1984: 233).

De ce fait, les activités d'éveil métalinguistique permettant d'amener les apprenants à s'interroger sur la forme et le fonctionnement de la L1 favoriseraient l'acquisition de la L2. À ce niveau, le rôle de l'éveil aux langues paraît crucial car "l'Éveil aux langues et, plus largement, les activités d'enseignement qui convoquent des ressources langagières multiples, poursuivent, entre autres, l'objectif de développer des compétences métalinguistiques chez les élèves concernant le fonctionnement du langage. 》 (Auger et Kervran 2013: 265).

De même, dans les situations d'acquisition d'une L2, des phénomènes psychologiques complexes de tension entre le désir d'intégration et la peur d'assimilation peuvent apparaître chez l'individu si L1 et L2 n'ont pas le même statut. C'est généralement le cas 
dans les situations de diglossie où l'une des langues est dévalorisée par rapport à l'autre. Ainsi, dans l'enseignement bilingue songhay-français du Mali, le français, reconnu comme langue d'expression officielle et formalisé par la constitution de 1992 dans son article 25 , a évidemment un statut social plus élevé que le songhay. Seule la valorisation systématique des deux langues permet à l'apprenant de sortir de cette double contradiction. Pour reprendre les termes de Bernabé (1999), L1 et L2 doivent être dans une relation de duo et non pas de duel.

La seconde partie de notre cadre théorique concerne les fonctions des interactions pédagogiques: les fonctions pragmatiques, les fonctions dialogiques et les fonctions didactiques.

Dans le cadre énonciatif, il existe trois fonctions pragmatiques fondamentales, à savoir l'assertion, la question et l'injonction. Elles constitueraient les actes basiques de l'interaction, en situation didactique, voire en toute situation où il y a échange de paroles. Ces principaux actes de langage se retrouvent dans toutes les langues. Ils renvoient respectivement à la proposition assertive, à la proposition interrogative et à la proposition impérative.

S'agissant de la fonction dialogique, nous nous limitons au dialogue scolaire qui obéit au schéma « initiative, réponse, évaluation » de Sinclair et Coulthard (1975). Ce schéma est exploité pour les situations didactiques par Celik et Mangenot (2004: 77) : " Consignes $=>$ Contributions répondant aux consignes $=>$ Feed-back $»$. Ainsi, l'évaluation est « consubstantielle des processus d'enseignement et d'apprentissage » (Huver et Springer 2011: 31). C'est pourquoi, il y a « des traces constantes d'activité évaluative » (ibid.) dans les interactions didactiques.

31 Concernant la fonction didactique, nous soulignons que le discours de la classe se construit à travers les interactions et les relations interpersonnelles entre le maittre et les apprenants (Bigot 2005). Les compétences s'acquièrent par le truchement des explications du maître et, dans l'enseignement-apprentissage bilingue spécifiquement, par celui de l'intégration comparative des enseignements linguistiques qui renforcent l'apprentissage de chaque langue, tout en favorisant le transfert des compétences acquises dans chacune d'entre elles. L'explication et la comparaison interlangues sont essentielles pour amener les apprenants à accomplir les tâches qui leur sont proposées par le maître.

\section{Méthodologie}

La présente étude privilégie en plus de l'observation directe de séquences de cours, l'approche quantitative et qualitative de collecte et d'analyse des données.

Nos données, recueillies en octobre 2011 à Gao dans l'une des écoles expérimentales d'enseignement bilingue au Mali, sont une partie de celles du projet plurinational, regroupant la France, le Burkina Faso, le Mali et le Niger, soutenu par l'OIF (Organisation Internationale de la Francophonie) et l'AUF (Agence Universitaire de la Francophonie), intitulé : «Transferts d'apprentissages $»^{1}$.

34 L'enquête a été menée auprès des élèves et maîtres des classes de $2^{\text {ème }}, 4^{\text {ème }}$ et $5^{\text {ème }}$ années de l'école fondamentale de « Gadèye B ». 
Tableau $\mathrm{N}^{\circ}$ 1: Nombre d'élèves constituant l'échantillon observé

\begin{tabular}{|l|l|l|l|l|}
\hline Classes & Effectif Total & Effectif des garçons & Effectif des filles & Sexe Enseignant \\
\hline $2^{\text {ème } a n n e ́ e ~}$ & 99 & 60 & 39 & F \\
\hline $4^{\text {è̀me } a n n e ́ e ~}$ & 78 & 36 & 42 & M \\
\hline $5^{\text {è̀me année }}$ & 53 & 24 & 29 & F \\
\hline
\end{tabular}

Source : Tableau reconstitué à partir de l'effectif des classes de $2^{e}, 4^{e}$ et $5^{e}$ années de l'école fondamentale de Gadèye B

Les âges des élèves se répartissent entre 6 et 14 ans. Les langues utilisées sont le songhay (L1) qui est la langue première des élèves, puis le français (L2) qui constitue leur langue seconde. Notre corpus comprend six séquences d'enregistrements vidéo d'une durée totale de 3 heures 50 minutes 38 secondes. Ainsi, nous avons filmé trois séquences de cours en calcul et en sciences d'observation respectivement dans les classes de $2^{\text {ème }}, 4^{\text {ème }}$ et $5^{\text {ème }}$ années fondamentales.

En plus, des enregistrements vidéo, notre corpus contient les photographies de dix productions écrites des élèves, sur les ardoises, dans les cahiers ; ainsi que trente photographies représentant la trace de ce qui est inscrit au tableau, des pages pertinentes du manuel, du cahier de préparation des maitres, des documents pédagogiques et des affiches dans les classes observées.

7 Nous avons choisi CLAN (Computerized Language Analysis) comme outil de transcription des séquences vidéo. C'est l'outil qu'a utilisé le projet CHILDES. Adopté par le projet "Transferts d'apprentissage", CLAN est une série de programmes informatiques pour traiter et analyser les données qui permet d'aligner parfaitement l'énoncé de la transcription avec le média visuel (Parisse 2014).

En vue de l'analyse des données, nous avons codé notre corpus par des lignes additionnelles commençant par des «\%m: " pour : «Analyse métalinguistique », en dessous de la ligne principale de la transcription. Nous avons codé les traces de comportement épilinguistique et les traces d'activité métalinguistique. Notre codage s'est effectué à deux niveaux : la trace de comportement épilinguistique codée par $\mathrm{E}$ et la trace d'activité métalinguistique codée par $\mathrm{M}$. Le deuxième niveau concerne: la position Énonciative (Assertion, Question, Injonction) = EA, EQ, EI; la fonction Dialogique (Initiative, Réponse, Evaluation) = GI, GR, GE et la fonction Didactique (Correction, Explication, Comparaison) $=\mathrm{TR}$, TE, TC. Nous avons utilisé les deux commandes de CLAN (FREQ et COMBO) pour étudier en détail tous les codages réalisés dans notre corpus et récupérer rapidement les résultats nécessaires à notre travail de recherche.

Le modèle théorique que nous avons choisi a exigé de nous une méthode de recherche principalement qualitative même si grâce aux codages des traces de comportement épilinguistique, d'activité métalinguistique et de gestes co-verbaux, nous avons pu procéder à des évaluations quantitatives. 


\section{Résultats}

Nous avons dénombré 30 traces de comportements épilinguistiques des élèves dans les séquences de calcul. Ces traces relèvent exclusivement de l'assertion et de la correction successivement au niveau de la position énonciative et de la fonction didactique. Parmi elles, 14 sont des réponses et 16 des évaluations au niveau dialogique. Les traces d'activités métalinguistiques des élèves dans les séquences de calcul atteignent 197 occurrences. Elles sont essentiellement des assertions (194), des évaluations (140) et des corrections (187). Toutefois, parmi elles, il y a des questions (3), des réponses (56) et des explications (10). Aucune d'elles ne relève de l'injonction et de la comparaison, une seule est une initiative.

Le graphique suivant présente la distribution des traces de comportements épilinguistiques et des traces d'activités métalinguistiques des élèves, dans les trois séquences de calcul, en fonction des contextes de l'énonciation, du dialogue et de l'activité didactique.

43 En sciences d'observation, les traces de comportements épilinguistiques des élèves sont plus nombreuses qu'en calcul. Sur les 76 occurrences que nous avons répertoriées, 75, au niveau énonciatif, sont des assertions ; 73, au niveau dialogique, des réponses et 45 , au niveau didactique, des explications. À ce dernier niveau, 23 occurrences relèvent de la correction.

44 Les occurrences de traces d'activités métalinguistiques listées dans les séquences de sciences d'observation sont au nombre de 168. En suivant l'ordre respectif de la position énonciative, de la fonction dialogique et de la fonction didactique, 165 cas apparaissent dans les assertions, 159 dans les réponses et 117 dans les explications.

Le diagramme ci-dessous laisse voir clairement, dans les trois contextes (position énonciative, fonction dialogique et fonction didactique), les traces de comportements épilinguistiques et d'activités métalinguistiques observées chez les élèves dans les séquences de sciences d'observation.

\section{Analyses}

\subsection{Analyse des comportements épilinguistiques des élèves}

Il est clair que l'élève se construit des représentations personnelles du fonctionnement de la langue. Il a sa propre vision du code langagier. Ce qui est très souvent en déphasage avec l'enseignement organisé qu'il suit à l'école. Les comportements épilinguistiques observés chez les élèves, dans les séquences de calcul, s'inscrivent dans ce contexte. La plupart d'entre eux se rapportent aux hésitations et aux erreurs commises dans le comptage.

Exemple $^{\circ}$ 1: Mson-A2-cal-L1-171011 


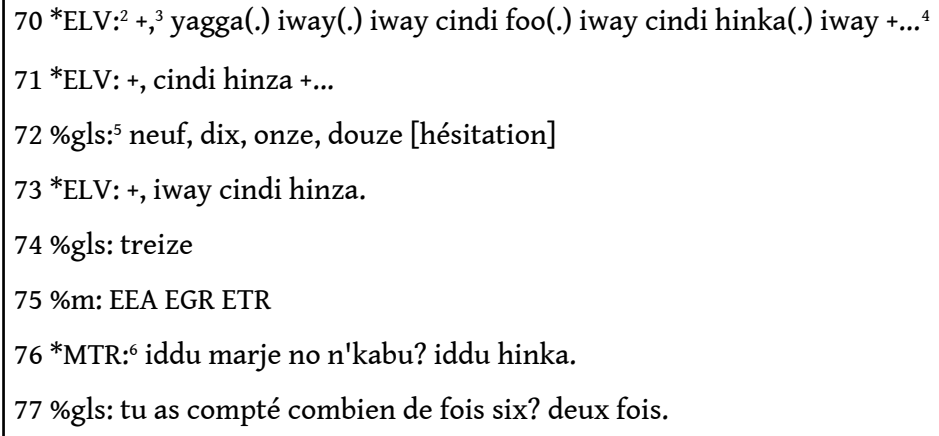

Dans cet exemple, la maîtresse, en faisant savoir à l'élève en songhay que deux fois six font douze, l'incite à s'apercevoir que son dénombrement ne doit pas atteindre treize qui est supérieur à douze.

Dans l'exemple qui suit, l'élève a de la peine à prononcer le mot « règle ».

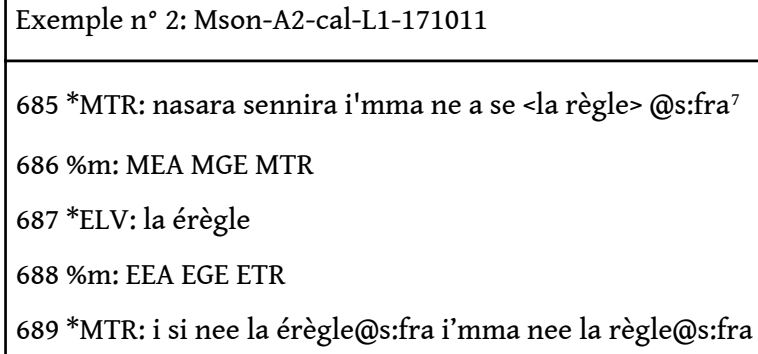

49 Il est très rare de trouver le $/ \mathrm{r} /$ en position initiale en songhay. Ce qui fait que l'élève qui découvre le français à l'école a du mal à prononcer les mots commençant par $/ \mathrm{r} /$. Il commence généralement par prononcer la voyelle qui suit le /r/ avant de lire le mot. Aussi est-il fréquent d'entendre des locuteurs du songhay prononcer « aradio » au lieu de "radio ». Le rôle de l'enseignant est aussi de nourrir l'activité épilinguistique de l'élève en enrichissant spontanément le milieu langagier dès que se présentent des circonstances qui le lui permettent. Il est important d'entraîner les élèves à lire des mots du français commençant par $/ \mathrm{r} /$.

En effectuant les opérations de calcul, il arrive de constater que l'élève s'imagine déjà en train de compter de l'argent.

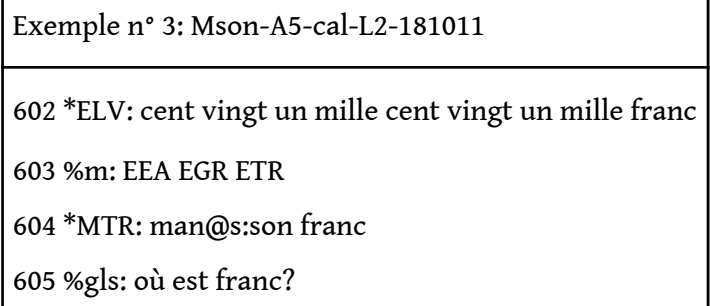

51 Ici, l'élève, en lisant les chiffres écrits au tableau, prononce le mot « franc » qui n'est inscrit nulle part devant lui. Il est à souligner que de nos jours, la monnaie utilisée au Mali est le franc CFA (Colonies Françaises d'Afrique) devenu le franc de la Communauté 
Financière Africaine depuis 1960. Le terme "franc » se dit dans ce pays dès lors qu'on prononce un chiffre se rapportant à l'argent.

Les comportements épilinguistiques dans les séquences de sciences d'observation relèvent le plus souvent des interférences linguistiques entre la L1 et la L2. Dans l'exemple qui suit, l'élève traduit le vocable songhay « dulloo » (la fumée, la vapeur) par « fumée ».

Exemple ${ }^{\circ}$ 4: Mson-A5-scienobs-L2-181011

600 *MTR: cinno i ga nee wo din se dulloo din kay ga diya cin no i ga nee a se? $\bullet 862742 \_868262 \bullet$

$601 \% \mathrm{gls}$ : comment on l'appelle cette vapeur que tu vois?

602 \% : MEQ MGE MTE

603 *ELV: fumée

604 \%m: EEA EGR ETE

605 *MTR: macin no i ga nee a se koyne kay mana tii fumée?

606 \%gls: qu'est-ce qu'on dit d'autre un terme différent de fumée?

607 \% : MEQ MGE MTE

608 *MTR: han@i cin no i ga nee a se koyne?

609 \%gls: comment on l'appelle encore?

610 \%m: MEQ MGE MTE

En songhay, c'est le même terme «dullu » qui désigne à la fois la fumée et la vapeur. Or en français, les deux vocables « fumée » et « vapeur » ont deux significations distinctes. L'élève sait traduire le mot « dullu » par « fumée », mais il ne connait probablement pas le mot « vapeur».

La comparaison entre L1 et L2 est nécessaire pour entretenir l'activité épilinguistique des élèves et la ramener à une activité métalinguistique.

Les comportements épilinguistiques des élèves se manifestent parfois dans les descriptions. Dans le cours de $5^{\text {ème }}$ année sur l'eau et ses différents états, les élèves tentent de décrire en L2 une petite quantité d'eau contenue dans un sachet en plastique qu'ils manipulent.

\begin{tabular}{|l|}
\hline Exemple n 5: Mson-A5-scienobs-L2-181011 \\
\hline 256 *ELV: l'eau est balancée \\
$257 \%$ m: EEA EGR ETR \\
{$[\ldots .]$.} \\
262 *ELV: l'eau est tremblée \\
$263 \%$ m: EEA EGR ETR
\end{tabular}

56 Ils ont sûrement voulu dire que l'eau est liquide, qu'elle est fluide ou qu'elle est gluante. Mais vu que leur répertoire linguistique est faible en français, ils n'ont pas su bien exprimer leur jugement. 


\subsection{Analyse des activités métalinguistiques des élèves}

\section{les élèves dans le comptage.}

Les élèves se servent de bâtonnets pour effectuer les dénombrements. La manipulation joue un rôle important dans le cours de calcul. Elle permet à l'élève de savoir comment apprendre et comment découvrir les choses de lui-même. Comme le postule Berdonneau (2006: 1), « apprendre les mathématiques, ce n'est pas mémoriser des règles ou des informations, mais s'entrainer à raisonner sur des objets abstraits, pour établir des propriétés sans avoir à recourir à l'expérience pour valider ses conclusions ». l'élève à quoi sert tout ce qu'il apprend à l'école.

60 Les traces d'activités métalinguistiques relevant de la composante métagraphique peuvent être analysées grâce aux photographies que nous avons prises en classe. A travers la photographie ci-dessous, nous constatons que les apprentissages acquis en L1 sont petit à petit transférés en L2. L'élève, qui a appris en songhay à connaître les chiffres et à compter depuis la $1^{\text {ère }}$ année, poursuit cet apprentissage en $2^{\text {ème }}$ année, cette fois en ayant l'équivalence de ses connaissances de la $1^{\text {ère }}$ année en français. Les inscriptions telles qu'elles sont affichées au tableau lui permettent de prendre conscience du fait que les chiffres dans les deux langues s'écrivent de la même façon. Mais leurs noms diffèrent selon la langue. 


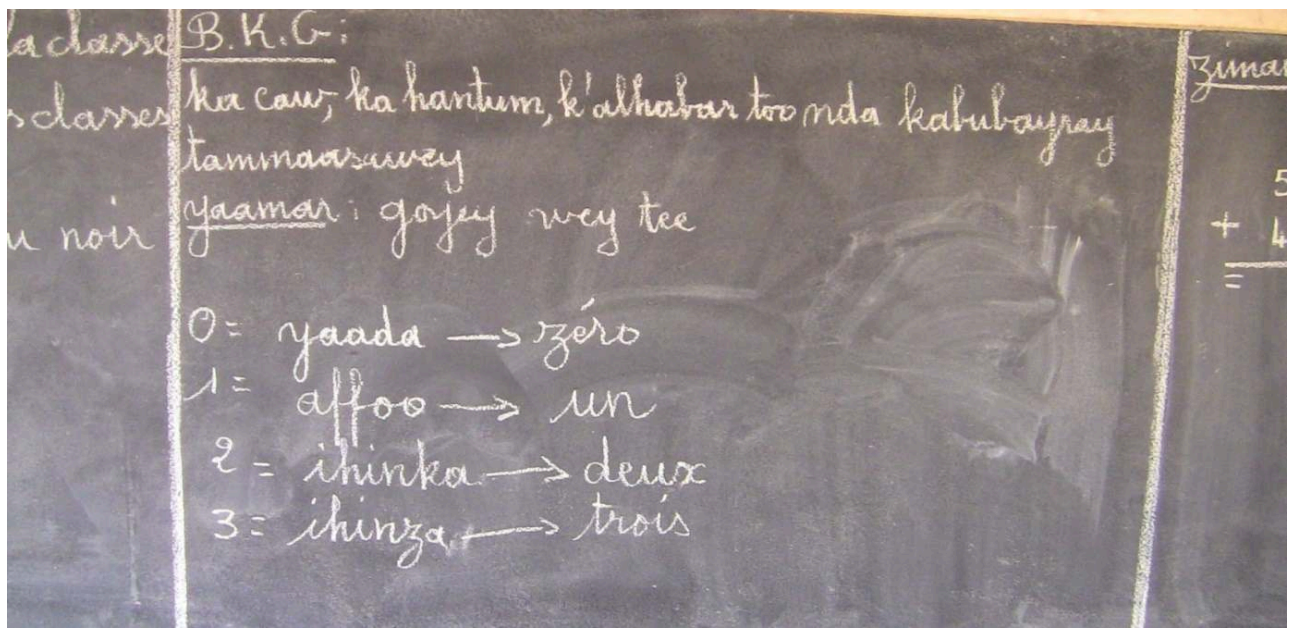

Photographie $\mathrm{N}^{\circ} 1$ : Chiffres inscrits au tableau

L'élève, qui est capable d'effectuer les opération en L1, va être forcément capable d'effectuer les mêmes opérations en L2 d'autant plus que les chiffres ont les mêmes graphies dans les deux langues.

traces d'activités métalinguistiques des élèves dans les séquences de sciences d'observation se rapportent aux verbalisations qu'ils accomplissent sur demande du maître. Elles relèvent essentiellement de la composante métalexicale ou métasémantique qui est relative aux connaissances sur les rapports entre signifiants et signifiés. Les élèves répondent aux sollicitations des enseignants en nommant des objets ou en donnant des exemples.

En $2^{\text {ème }}$ année, plusieurs élèves sont interrogés pour donner chacun un exemple de fruit.

\begin{tabular}{|l|}
\hline Exemple n 7: Mson-A2-scienobs-L1-211011 \\
46 *MTR: un fruit comme exemple fruit@s:fra foo noo yane ya diya \\
47 \%gls: donnez-moi un exemple de fruit \\
48 \%m: MEQ MGI MTE \\
49 *ELV: leemurba \\
50 \%gls: orange \\
51 \%m: MEA MGR MTE \\
[...] \\
97 *ELV: banaana \\
98 \%gls: banane \\
$99 \%$ m: MEA MGR MTE \\
[...] \\
426 *ELV: goyave@s:fra \\
427 \%m: MEA MGR MTE
\end{tabular}


L'usage de la L2 par les élèves est essentiellement destiné à répondre aux sollicitations visant à donner un exemple, à nommer un objet ou une partie de l'objet. Les sollicitations liées à la description sont généralement répondues en L1.

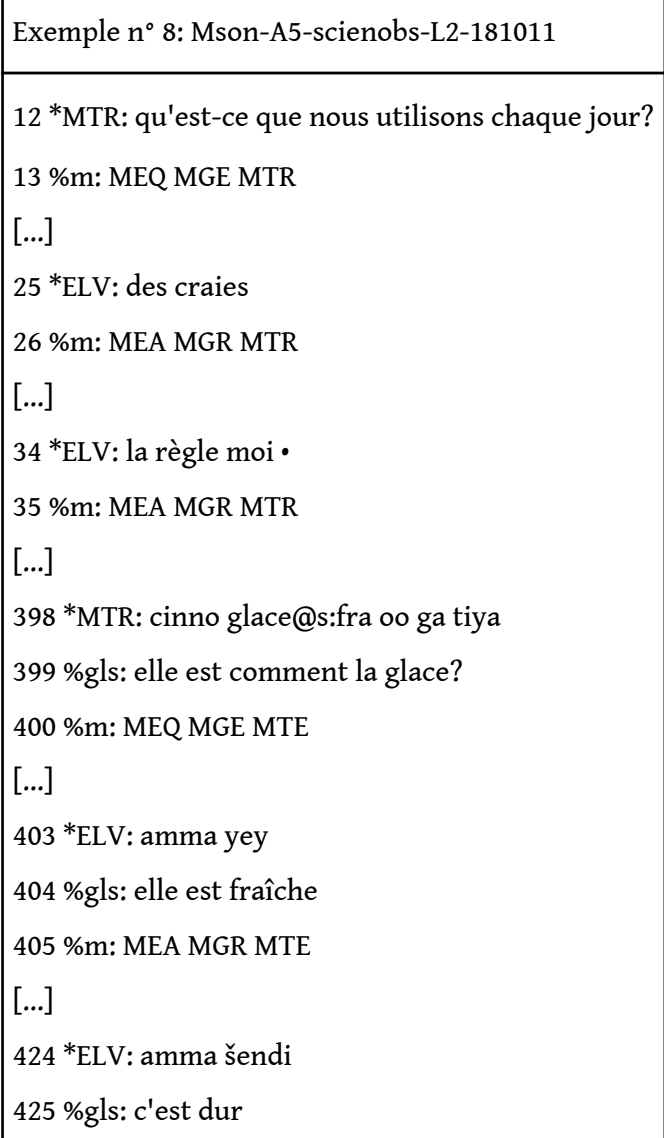

Le medium d'enseignement utilisé est la L1, d'où l'aisance des élèves dans l'expression. Nous pouvons donc assurer que l'utilisation de la langue maternelle de l'élève permet d'établir des ponts entre les connaissances de l'école et celles du dehors autrement dit celles apprises en famille. L'intérêt que l'école accorde à la culture que l'élève acquiert en famille ne peut que motiver celui-ci à vouloir en apprendre davantage, ce qui concourt à sa réussite scolaire.

\section{Conclusion}

Les comportements épilinguistiques des élèves sont récurrents dans les séquences que nous avons analysées. Ces comportements nécessitent la mise en place par l'enseignant d'activités d'éveil aux langues qui ont pour rôle de permettre aux enfants de convertir leur comportement épilinguistique en activité métalinguistique. Par exemple, le fait que certains élèves n'arrivent pas à prononcer le son $[r]$ devait être l'occasion pour le maître de les entraîner à lire des mots du français commençant par [r] et à faire une comparaison avec le songhay. De même, le terme de L1 « dullu » qui se réfère en L2 à la fois à la "fumée " et la "vapeur», a fait l'objet de plusieurs comportements 
épilinguistiques des élèves. La nécessité d'une activité d'éveil aux langues s'impose pour permettre aux apprenants de saisir à ce niveau la nuance entre L1 et L2.

Les traces d'activités métalinguistiques, quant à elles, en calcul, sont nombreuses dans le comptage et dans le maniement des objets. Dans les séquences de sciences d'observation, les activités métalinguistiques effectuées par les élèves ont trait généralement à la dénomination des objets étudiés et à la recherche d'exemples.

Nos analyses ont montré que l'apprenant de l'école bilingue, qui a sa propre vision des codes peut, à partir de comportements et d'activités épilinguistiques et métalinguistiques, acquérir des apprentissages pouvant être transférés d'une langue à l'autre et d'une discipline à l'autre.

\section{BIBLIOGRAPHIE}

Auger N. \& Kervran M. (2013). Répertoires langagiers, interactions didactiques et co-construction du langage à l'école primaire : l'exemple de la discussion. In Violaine Bigot, Aude Bretegnier et Marité Vasseur (éds.), Vers le Plurilinguisme? Vingt ans après Paris : les archives contemporaines. 265-273.

Baker C. (1993(1996)). Foundations of Bilingual Education and Bilingualism. Clevedon :, Multilingual Matters Ltd.

Belloncle G. (1984). La question éducative en Afrique. Paris : Karthala..

Berdonneau C. (2006)/ De l'importance des gestes pour l'apprentissage des concepts mathématiques, $4 \mathrm{p}$. [En ligne] Disponible sur: http://ecoles.ac-rouen.fr/circ_dieppe_est/outils/maternelle/ doc_maternelle/berdonneau02.pdf.

Bernabé J. (1999). La relation créole-français : duel ou duo ? Implications pour un projet scolaire. In Langues et cultures régionales de France, Etat des lieux, enseignement, politiques. Paris : L'Harmattan, coll. « Logiques sociales ». 35-52.

Bialystok E., Craik Fergus I.M., Luk G. (2012). Bilingualism: consequences for mind and brain. In Trends in Cognitive Sciences 16. 240-250.

Bigot V. (2005). Quelques questions de méthodes pour une recherche sur la construction de la relation interpersonnelle en classe de langue : primauté des données et construction de savoirs. Le Français dans le monde, Recherches et applications. 42-53.

Candelier M. (2003). Evlang - l'éveil aux langues à l'école primaire - Bilan d'une innovation européenne. Bruxelles : De Boek - Duculot..

Celik C. \& Mangenot F. (2004). La communication pédagogique par forum : caractéristiques discursives. Les Carnets du Cediscor 8. 75-88.

Culioli A. (1968). La formalisation en linguistique. Cahiers pour l'analyse 9. 106-117.

Cummins J. (1984). Bilingualism and special education: Issues in assessment and pedagogy. Clevedon : Multilingual Matters Ltd.. 
Demont E., Gaux C. \& Gombert J.-E. (2006). Bilan métalinguistique. In Les bilans de langage et de voix. Paris : Masson.

Doumbia A. T. (2000). L'enseignement du bambara selon la pédagogie convergente au Mali : théorie et pratiques. Nordic Journal of African Studies 9/3. 98-107

Gombert J.-E. (1990). Le développement métalinguistique. Paris : PUF.

Huver E. \& Springer C. (2011). L'évaluation en langues. Paris : Didier.

Kanta T., Blanca E. \& Rey V. (2006). La conscience phonologique et l'apprentissage d'une langue seconde, Skholê hors-série n¹. 53-58.

McHardy S. \& Coulthard M. (1975). Towards an Analysis of Discours : the English Used by Teachers and Pupils. Londres : Oxford University Press.

Noyau C. (2014). Construction de connaissances en L1 et en L2 : les transferts de connaissances en sciences d'observation. Recherches Africaines 14 «Transferts d'apprentissages ». Actes des Journées internationales de novembre 2013 (Ouagadougou, Burkina Faso) du projet AUF et OIF. 117-138.

Parisse C. (2014). Méthodologie de la recherche sur corpus pour l'étude des interactions dans les écoles bilingues. Recherches Africaines 14 « Transferts d'apprentissages. Actes des Journées internationales de novembre 2013 (Ouagadougou, Burkina Faso) du projet AUF et OIF. 9-20.

Werner L. (1949). Speech development of a bilingual child, New-York : Academic Press.

\section{NOTES}

1. Projet «Transferts d'apprentissage et mise en regard des langues et des savoirs à l'école bilingue : des points de vue de l'élève aux activités de classe ", OIF et AUF (2011-2014), rassemblant des équipes de 4 pays : Burkina Faso, Mali, Niger et France. URL: http:// modyco.inist.fr/transferts/

2. *ELV : énoncé produit par un élève.

3. +, : énoncé complétant un énoncé précédent produit par le même locuteur.

4. +...: énoncé en suspend.

5. \%gls : ligne secondaire contenant une glose en français du sens global des énoncés.

6. *MTR : énoncé produit par le maître.

7. @s:fra: indique qu'un mot n'est pas en L1 mais en L2.

8. (.) : pause courte.

\section{RÉSUMÉS}

Notre recherche porte sur la place du métalinguistique dans le contexte du bilinguisme scolaire où les élèves sont sensibilisés aux spécificités de l'univers du langage à partir d'un certain nombre d'activités d'observation et de réflexion portant sur des faits de langue. Pour expliquer les comportements cognitifs vis-à-vis du langage, Gombert (1990) propose de classer les activités de type métalinguistique en deux catégories : les activités épilinguistiques et les activités métalinguistiques. Etant donné que les situations d'enseignement dans les disciplines non- 
linguistiques sont les mieux indiquées pour étudier chez les élèves bilingues le développement du langage en deux langues, il est important de chercher à savoir comment le métalinguistique se manifeste dans ces disciplines. A partir des résultats issus du codage de six enregistrements vidéo, en mathématiques et en sciences d'observation (physique, chimie et sciences naturelles), effectués dans trois classes d'une école bilingue songhay-français du Mali, nous avons analysé les comportements épilinguistiques et les activités métalinguistiques des élèves.

Our research refers to the place of metalinguistic in the context of bilingual school where pupils are aware of the specifics of the language universe from a certain number of observation and reflection activities on the language facts. To explain cognitive behavior in regard to language, Gombert (1990) proposes to classify the type of metalinguistic activities into two categories: epilinguistic activities and metalinguistic activities. Given that teaching situations in nonlanguage subjects are best shown in studying in bilingual pupils language development in two languages, it is important to investigate how the metalinguistic occurs in these disciplines. Based on the results from the coding of six video recordings in mathematics and in sciences of observation (Physics, chemistry and natural sciences) made in three classes with a bilingual school Songhay-French in Mali, we analyzed the epilinguistic behavior and metalinguistic activities of students.

\section{INDEX}

Mots-clés : métalinguistique, épilinguistique, disciplines non-linguistiques, songhay, français, école bilingue

Keywords : metalinguistic, epilinguistic, non- linguistic disciplines, Songhai, French, bilingual school

\section{AUTEUR}

\section{ZAKARIA NOUNTA}

Université de Ségou (Mali) Faculté des sciences sociales Filière : communication des organisations 